\title{
12. SOME RESULTS OF INVESTIGATIONS OF INSTANTANEOUS METEOR PHOTOGRAPHS
}

\author{
P. B. BABADŽANOV \\ and \\ E. N. KRAMER \\ (Astrophysical Institute of the Tadžik \\ Academy of Sciences, Dušanbe, U.S.S.R.) \\ (University Observatory, Odessa, \\ U.S.S.R.)
}

A BST R A C T

On the basis of photographic observations made by the method of instantaneous exposure, the wake and terminal blending phenomena were investigated. The first of these phenomena is explained by fragmentation, and the second by the recombination of meteor atoms and ions. The successive disintegrations of the original meteor body were also studied.

Special photographic observations of meteors have been carried out since 1964 at Dušanbe and Odessa, using the method of instantaneous expasure. The aim of these observations is to study the evaporation, luminescence and fragmentation processes.

The nature of meteor phenomena, and particularly the meteor form and size at a given moment, are determined to a considerable degree by fragmentation. The optical system used makes possible the observation of objects with linear sizes exceeding 200-500 m at distances of 100-150 km. But the fast motion of the meteor image on the film spreads the details of the meteor phenomena. Therefore instantaneous meteor photographs are most attractive. The meteor displacement for an exposure of $10^{-3}$ $\mathrm{sec}$ is indeed ten times less than the size of the meteor, even for velocities $60-70 \mathrm{~km} / \mathrm{sec}$, and for an exposure of $10^{-4} \mathrm{sec}$ it falls inside the limits of the resolving power of the optical system. Thus, the method of instantaneous exposure allows us to obtain a 'pure' meteor image, irrespective of the meteor velocity.

About 10000 instantaneous images of more than 100 meteors have been obtained up to the present. The photographs described below have been obtained with a camera of focal length $F=750 \mathrm{~mm}$ and focal ratio $f=1: 3 \cdot 5$. A rotating shutter was placed in front of the film, i.e. in the close vicinity of the focal plane. This permits us to obtain meteor photographs with exposures of $0.0006 \mathrm{sec}$ every $0.02 \mathrm{sec}$; a panchromatic emulsion with sensitivity 1300 units GOST (approximately 350-400 ASA) was used.

The study of the instantaneous meteor images leads to the following classification:

Type I - a 'dotted' meteor image;

Type II - a coma in the form of a sharp projection behind the 'dotted' meteor image; 
Type IIa - an image, sharp on both sides, the longitudinal size being distinctly visible;

Type III - a meteor consisting of a head and a tail, reminiscent of a penumbra;

Type IV - a distinctly visible disk and a long tail.

From all photographed meteors some of the most representative ones were selected. In order to estimate the meteor's deviation from a point source, lengths of the images along the meteor trajectory were measured. In this case photographic diffusion was excluded and the displacement of the meteor images during the exposure was taken into account. Results of this measurement are given in Table 1.

Table 1

\begin{tabular}{|c|c|c|c|c|c|c|c|c|}
\hline $\begin{array}{l}\text { Meteor } \\
\text { No. }\end{array}$ & $\mathrm{Da}$ & ite & Shower & $\begin{array}{c}v^{v} \\
(\mathrm{~km} / \mathrm{sec})\end{array}$ & $\begin{array}{c}\eta \times 10^{4} \\
(\mathrm{sec})\end{array}$ & $\begin{array}{c}\tau \times 10^{4} \\
(\mathrm{sec})\end{array}$ & $\stackrel{l}{l}$ & Type \\
\hline 2 & & Aug. & Perseid & 60 & 45 & 9 & 55 & II \\
\hline 3 & 6 & ,, & Perseid & 58.9 & 50 & 10 & 60 & III \\
\hline 7 & 6 & , & Perseid & $61 \cdot 7$ & 70 & 14 & 85 & III \\
\hline 13 & 8 &, & & $24 \cdot 6$ & 756 & 151 & 370 & IV \\
\hline 16 & 9 &, & & 30 & 193 & 39 & 120 & III \\
\hline 22 & 11 & , & Perseid & 60 & 135 & 27 & 160 & III \\
\hline 28 & 11 &, & Perseid & 60 & 87 & 17 & 100 & III \\
\hline 29 & 11 & , & Perseid & 60 & 123 & 25 & 150 & III \\
\hline 30 & 10 &, & Perseid & 60 & 15 & 3 & 18 & II \\
\hline 31 & 10 & , & & 34.2 & 101 & 20 & 120 & III \\
\hline 34 & 12 & . & & 60 & 65 & 13 & 80 & III \\
\hline 38 & 10 & , & & 59.7 & 101 & 20 & 120 & Ila \\
\hline 39 & 12 & , & & $24 \cdot 6$ & $>2500$ & $>500$ & $>1200$ & IV \\
\hline 49 & 13 & , & & 19.4 & 215 & 43 & 260 & III \\
\hline
\end{tabular}

The successive columns in Table 1 contain: (1) the meteor number; (2) the day and the month of 1964 ; (3) the meteor identification; (4) the velocity $v$ - for seven meteors, Nos. 3, 7, 13, 31, 38, 39, 49 the velocity was calculated on the basis of two-station observations, for the identified Perseids and No. 34 a velocity of $60 \mathrm{~km} / \mathrm{sec}$ was assumed, and for No. 16, the radiant of which is near the ecliptic, a velocity of $30 \mathrm{~km} / \mathrm{sec}$ was assumed; (5) the maximum value of the ratio $(\eta)$ of the length of the meteor image to the distance between successive images; (6) the time $(\tau)$ required for passing of the maximum meteor length (the luminescence duration in maximum light); (7) the maximum length $(l)$ in metres: $l=0.02 v \eta$; (8) the types of the instantaneous images.

Practically everywhere the lengths of the meteor images are considerably larger than the resolving power of the camera and the measuring errors. The average lengths of the images of types II and III lie in the range $50-150 \mathrm{~m}$. This corresponds to fast meteors and particularly to the Perseids. On the instantaneous photographs the bright meteors usually have a bright head and a faint luminous tail. At the points 
outside an abrupt light increase the tail varies little in length, corresponding to the wake (W) phenomenon on usual meteor photographs (Jacchia et al., 1965). The tail length increases during every abrupt brightness rise and decreases during every abrupt brightness drop. The tail length may be larger than the interval between single instantaneous images, and intervals then fill up, corresponding to the phenomenon of terminal blending (Jacchia et al., 1965).

For the fragmentation case the tail length is determined by the displacement of the luminous fragments relative to the parent meteor body. But the observable meteor length may be due to radiation from a given point, which continues during a comparatively large time interval. In such a case the tail length is determined by the duration of luminosity at the given point.

The intensity distribution of meteor No. 31 (Table 2) is plotted in Figure 1. Such a distribution is observed in most of the instantaneous images of this meteor. The tail length of this meteor changes little and therefore the tail may be classified as a wake (W). It was shown by Babadžanov and Kramer (1965) that luminosity of the

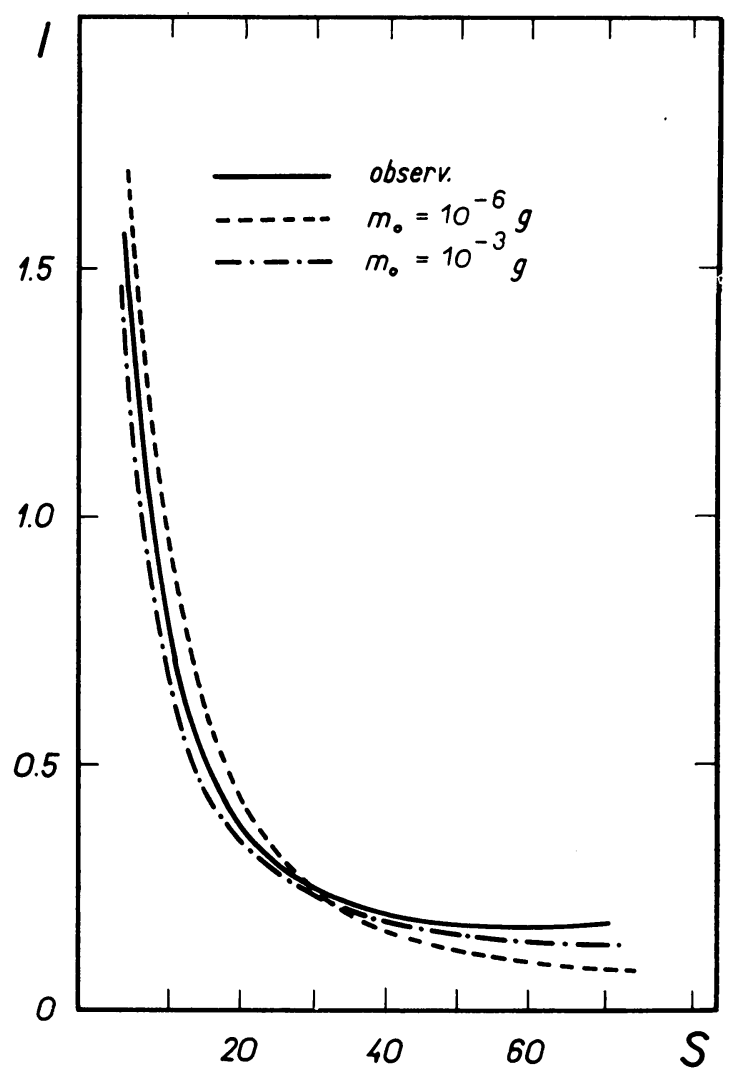

FIG. 1. The observed and computed intensity distribution in the tail of meteor No. 31 
Table 2

\begin{tabular}{|c|c|c|c|c|c|c|}
\hline \multirow{2}{*}{$\begin{array}{c}\text { Meteor } \\
\text { No. }\end{array}$} & \multirow[t]{2}{*}{ Date } & \multicolumn{2}{|c|}{$\begin{array}{c}\text { Radiant } \\
1950 \cdot 0\end{array}$} & \multirow{2}{*}{$\begin{array}{l}\text { Velocity } \\
(\mathrm{km} / \mathrm{sec})\end{array}$} & \multirow{2}{*}{$\begin{array}{l}\text { Altitude of the } \\
\text { measured } \\
\text { instantaneous } \\
\text { image }(\mathrm{km})\end{array}$} & \multirow{2}{*}{$\begin{array}{l}\text { Radiation intensity } \\
\text { at the head } \\
\text { of the instantaneous } \\
\text { image }(\mathrm{erg} / \mathrm{sec})\end{array}$} \\
\hline & & $\alpha$ & $\delta$ & & & \\
\hline 13 & 1964 Aug. 8 & $284 . .1$ & 45.6 & $24 \cdot 6$ & $80 \cdot 3$ & $1.15 \times 10^{12}$ \\
\hline 31 & 1964 Aug. 10 & $305: 7$ & 74.5 & $34 \cdot 2$ & $87 \cdot 7$ & $2.70 \times 10^{11}$ \\
\hline 39 & 1964 Aug. 12 & $283: 9$ & $46: 2$ & $24 \cdot 6$ & $84 \cdot 4$ & $1.12 \times 10^{12}$ \\
\hline
\end{tabular}

tails of this type cannot be explained by radiation of excited atoms evaporated directly from the surface of the meteor body (Halliday, 1958). We attempted to apply the procedure developed by McCrosky (1958) to meteor No. 31. In accordance with this procedure the wake luminescence is connected with quasi-continuous fragmentation of the meteor body (Levin, 1961), and with radiation of excited atoms evaporated from the surface of dust particles formed in consequence of fragmentation. The dust displacement relative to the parent body is determined by the formula

$$
S=v_{0} t-\frac{H^{*}}{\cos Z} \ln \left\{\frac{E_{i}\left(\begin{array}{l}
\sigma \\
6
\end{array} v_{0}^{2}\right)-E_{i}\left(\begin{array}{l}
\sigma \\
6
\end{array} v^{2}\right)}{2 \Gamma A \delta^{-2 / 3} H^{*} \rho_{0} e^{-\frac{\sigma}{6} v_{0}^{2}}} m_{0}^{1 / 3} \cos Z+1\right\},
$$

where $v_{0}$ is the velocity of the meteor body at the moment of fragmentation; $m_{0}$, the mass of the fragment at the moment of its breaking from the surface of the meteor body; $\sigma$, the ablation coefficient

$$
\sigma=\frac{\Lambda}{2 \Gamma Q}
$$

$\Lambda$, the heat transfer factor; $\Gamma$, the drag coefficient; $Q$, the heat of vaporisation; $A$, the shape factor; $\delta$, the density of the fragment; $Z$, zenith distance of the radiant; $H^{*}$ and $\rho_{0}$, the scale height and the air density respectively at the height of fragmentation (it is assumed that the corresponding atmospheric layer is isothermal); $t$, the time of the lag, corresponding to the change of the dust velocity from $v_{0}$ to the fixed value $v$.

From Equation (1) it follows that with decrease of $m_{0}$ the dust displacement (and consequently the tail length) increases.

In the meteor tail the total luminous intensity $(\mathrm{erg} / \mathrm{sec})$ of dust is determined by the formula

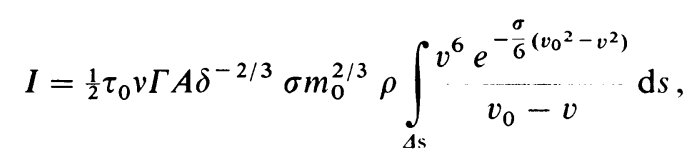


where $v$ is the velocity of meteor sputtering (i.e. number of particles breaking from the meteor-body surface per unit time); $\tau_{0}$, luminosity factor; $\rho$, air density at the altitude where the tail is observed; $\Delta s$, integrating interval, read from the fixed $s$ and corresponding to the exposure time $\Delta t=1 / 1800 \mathrm{sec}$ for the equipment used.

Theoretical curves calculated by McCrosky's method (with allowance for photographic diffusion) are also given in Figure 1 for values $m_{0}=10^{-6} \mathrm{~g}$ (circles) and $m_{0}=10^{-3} \mathrm{~g}$ (crosses). The observations are in good agreement with the theory for the case when the masses of the fragments are larger than $10^{-3} \mathrm{~g}$. For comparison it should be noted that McCrosky has obtained a satisfactory agreement of the theory with observations at $m_{0}=10^{-6}-10^{-4} \mathrm{~g}$. But he assumes that $\sigma=10^{-12}$, while we use the value $\sigma=3 \times 10^{-12}$. Our choice is determined by Equation (2). For dust motion the heat transfer coefficient may be taken equal to 1 , since in such a case it coincides with the accommodation coefficient (Levin, 1956). For the resistance coefficient the value $\Gamma=2$ was adopted. This may be the maximum possible value of $\Gamma$ for the dust motion. For $Q=8 \times 10^{10}$ we obtain $\sigma=3 \times 10^{-12}$. This value appears to be the maximum for the dust motion. Using this value of $\sigma$, McCrosky should obtain for $m_{0}$ values close to ours, although the pre-atmospheric mass of the meteor body was only 0.052 g. Therefore, for the explanation of wake luminescence McCrosky's theory requires a considerably larger mass for the fragments. This is in contradiction with the assumption of quasi-continuous fragmentation. Black-body radiation may change the form of the light curve. But thermal radiation affects meteor ablation and deceleration only in the case of sufficiently small sizes of particles at large altitudes and not too large velocities.

In Figure 2 the photograph of meteor No. 39 is presented (see Table 2). Its trajec-

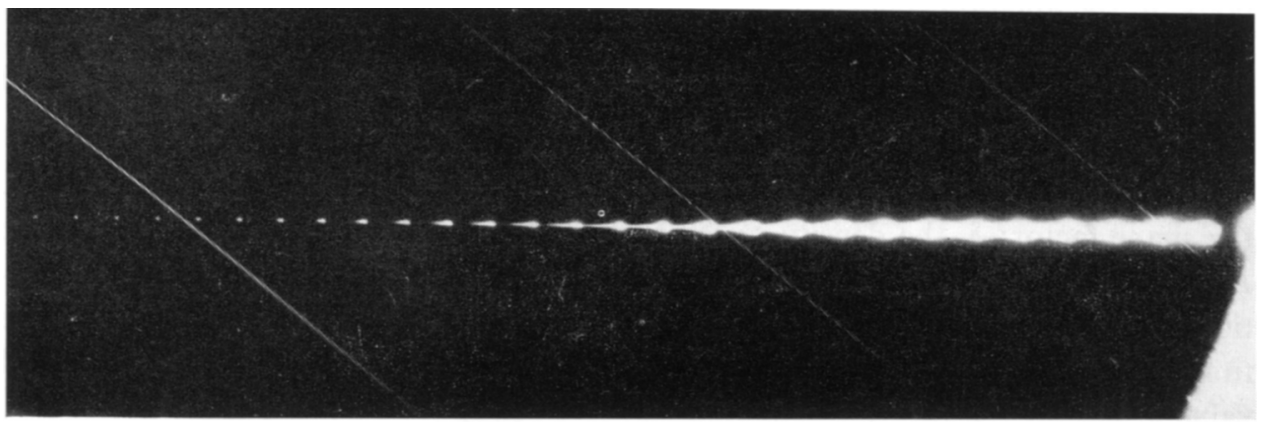

FiG. 2. Meteor No. 39 recorded by the method of instantaneous exposures.

tory was calculated on the basis of double-station photographic observations. The meteor appeared at the altitude of $103 \mathrm{~km}$ and disappeared at the altitude of $80 \mathrm{~km}$. Its mean velocity was $24.6 \mathrm{~km} / \mathrm{sec}$. There was a bright prolonged flare, which was brighter than mag. -10 . The spectrum of this meteor was obtained with a spectral camera. The bright sodium $\mathrm{D}$ line and fairly weak $\mathrm{H}$ and $\mathrm{K}$ lines of ionized calcium 
appear in it. This meteor left a persistent ionized train, which was visible more than $1 \mathrm{~min}$. Results of the measurement of the length of the meteor from instantaneous photographs are given in Table 3, where $d$ is the width of the meteor-head images. At the beginning of the trajectory the instantaneous meteor images are dotted. The tail appears later and increases continuously up to the burst, where the tail image is

Table 3

$\begin{array}{rrrrrrrr}N & d(\mathrm{~mm}) & \eta & l(\mathrm{~m}) & N & d(\mathrm{~mm}) & \eta & l(\mathrm{~m}) \\ 1 & 0.035 & 0.016 & 8 & 15 & 0.154 & 0.393 & 193 \\ 2 & 0.042 & 0.015 & 7 & 16 & 0 \cdot 152 & 0.490 & 241 \\ 3 & 0.042 & 0.014 & 7 & 17 & 0 \cdot 169 & 0.557 & 274 \\ 4 & 0.042 & 0.014 & 7 & 18 & 0 \cdot 177 & 0.701 & 345 \\ 5 & 0.040 & 0.014 & 7 & 19 & 0.196 & 0.769 & 378 \\ 6 & 0.049 & 0.023 & 11 & 20 & 0.197 & 0.965 & 475 \\ 7 & 0.056 & 0.029 & 14 & 21 & 0.221 & 1.000 & 492 \\ 8 & 0.067 & 0.030 & 15 & 22 & 0 \cdot 221 & 1.080 & 531 \\ 9 & 0.067 & 0.031 & 15 & 23 & 0.622 & 1.200 & 590 \\ 10 & 0.089 & 0.067 & 33 & 24 & 0.670 & 1.371 & 674 \\ 11 & 0.087 & 0.086 & 42 & 25 & 0.715 & 1.363 & 670 \\ 12 & 0.102 & 0.146 & 72 & 26 & 0.729 & 1.577 & 776 \\ 13 & 0.120 & 0.197 & 97 & 27 & 0.764 & 2.558 & 1258 \\ 14 & 0.134 & 0.253 & 124 & \cdots & & & \end{array}$

superimposed over the previous image. The main part of the flare is outside the frame of the photograph. The maximum length which could be measured exceeds $1.2 \mathrm{~km}$. This corresponds to an afterglow duration of $0.05 \mathrm{sec}$.

Both observed and theoretical variation curves of the linear intensity for the tail of meteor No. 39 are given in Figure 3 for $m_{0}=10^{-6} \mathrm{~g}$ and $m_{0}=10^{-3} \mathrm{~g}$. Computations show that the fragmentation theory does not agree with observations for any possible values of $m_{0}$. It should be noted that in the case of meteor No. 39 we deal with terminal blending (B). Thus, it is not possible to explain the phenomenon of terminal blending by fragmentation only.

Several instantaneous images of meteor No. 13 (see Table 2) are presented in Figure 4. The meteor appeared at the altitude of $90 \mathrm{~km}$ and disappeared at the altitude of $76 \mathrm{~km}$. Its mean velocity was $24 \cdot 6 \mathrm{~km} / \mathrm{sec}$. It belongs to the same meteor association as meteor No. 39.

Results of the measurement of the length of all its images are given in Table 4.

The instantaneous images of meteor No. 13 (Figure 4) may be compared with its normal (non-shutter) photograph (Figure 5). The meteor flares are distinctly visible on the normal photograph. They correspond to the instantaneous meteor-length increase. At the brightest flare the instantaneous meteor length is approximately equal to $370 \mathrm{~m}$, corresponding to an afterglow time at a given point of $\tau=0.016 \mathrm{sec}$. By that time the atoms, vaporised immediately from the meteor-body surface, lose nearly 


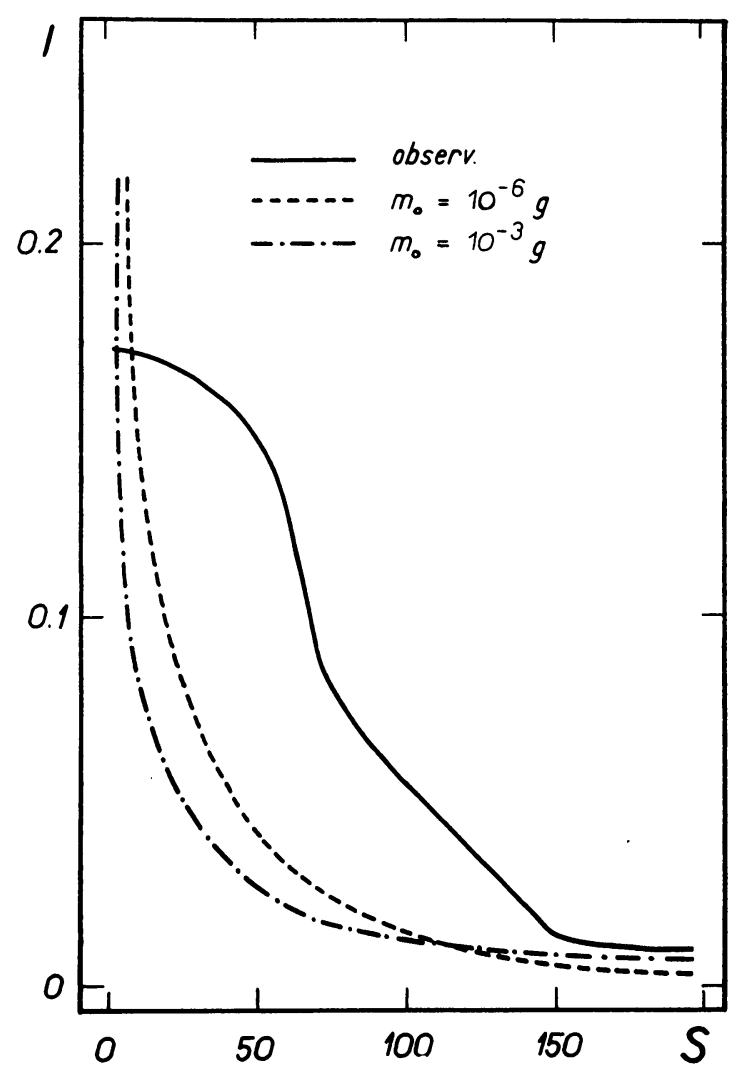

FIG. 3. Variation curves of the linear intensity for the tail of meteor No. 39.

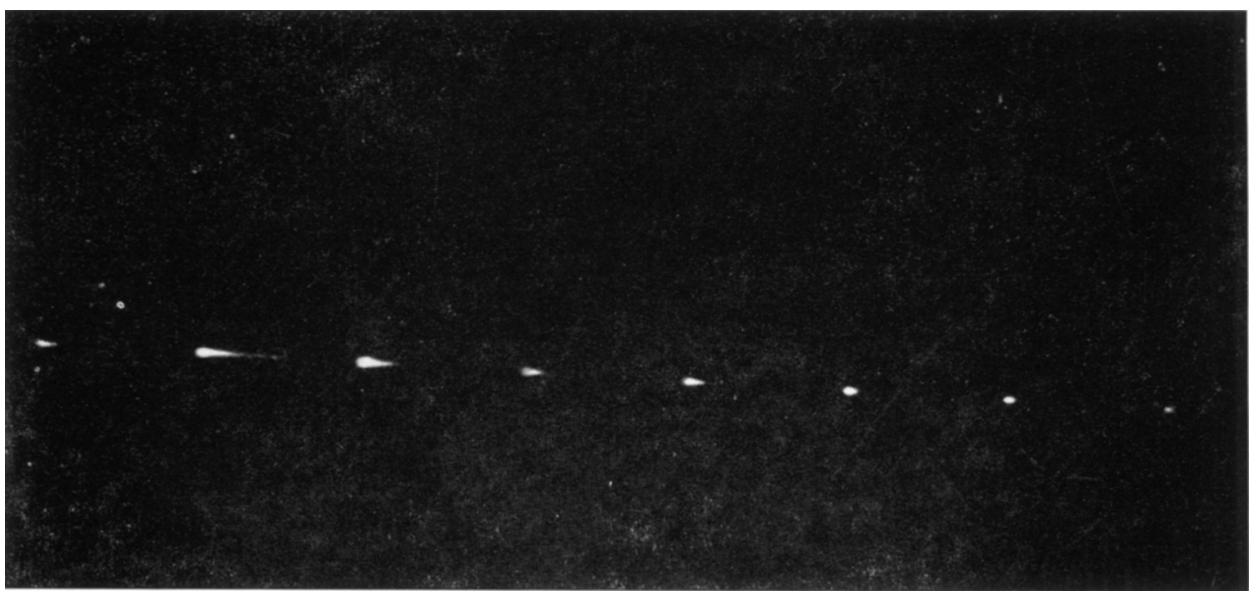

Fig. 4. A series of instantaneous exposures of meteor No. 13. 


\section{Table 4}

$\begin{array}{rrrrrrrr}N & d(\mathrm{~mm}) & \eta & l(\mathrm{~m}) & N & d(\mathrm{~mm}) & \eta & l(\mathrm{~m}) \\ 1 & 0.042 & 0.030 & 15 & 13 & 0.044 & 0.180 & 88 \\ 2 & 0.038 & 0.075 & 37 & 14 & 0.048 & 0.225 & 111 \\ 3 & 0.045 & 0.042 & 21 & 15 & 0.070 & 0.100 & 49 \\ 4 & 0.042 & 0.077 & 38 & 16 & 0.095 & 0.180 & 88 \\ 5 & 0.040 & 0.077 & 38 & 17 & 0.118 & 0.280 & 138 \\ 6 & 0.042 & 0.168 & 83 & 18 & 0.119 & 0.269 & 132 \\ 7 & 0.045 & 0.112 & 55 & 19 & 0.133 & 0.318 & 156 \\ 8 & 0.051 & 0.099 & 49 & 20 & 0.164 & 0.756 & 372 \\ 9 & 0.041 & 0.052 & 26 & 21 & 0.130 & 0.322 & 158 \\ 10 & 0.042 & 0.147 & 72 & 22 & 0.080 & 0.086 & 42 \\ 11 & 0.048 & 0.206 & 101 & 23 & 0.040 & 0.061 & 30 \\ 12 & 0.035 & 0.249 & 122 & & & & \end{array}$

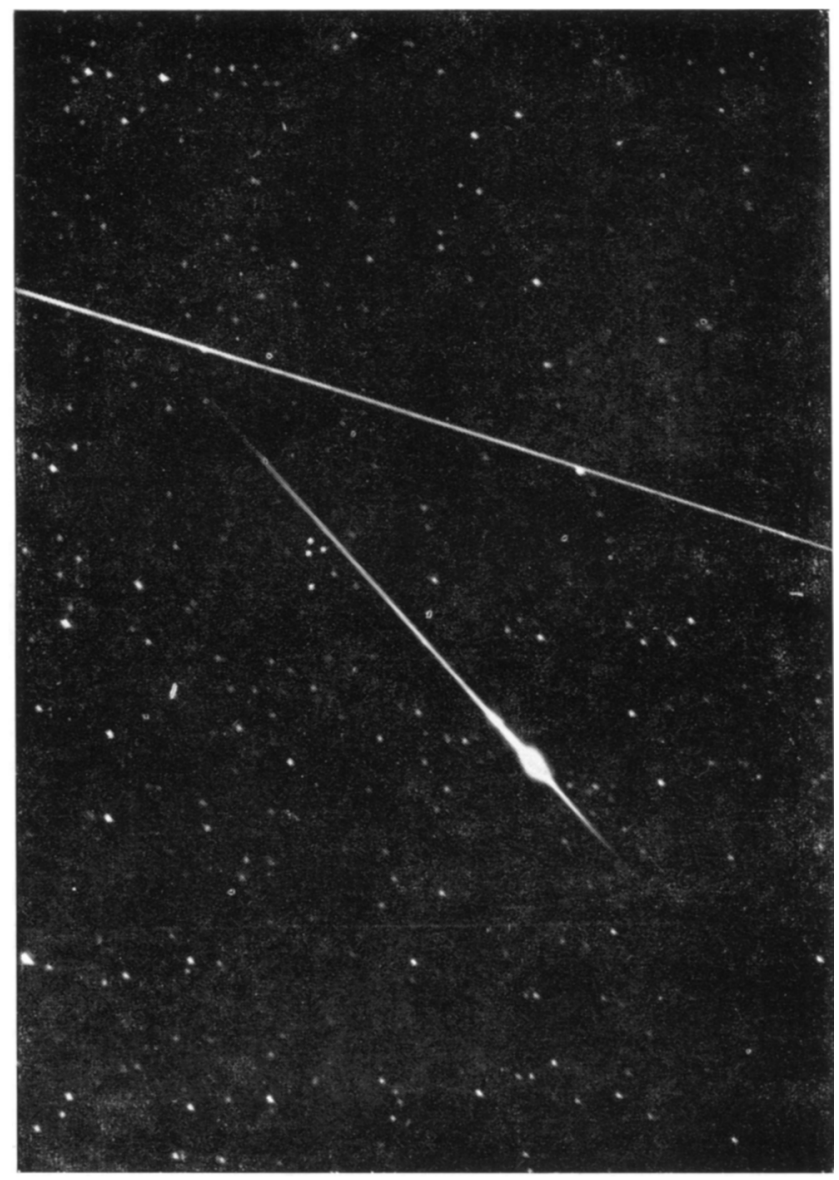

Fig. 5. A direct photograph of meteor No. 13. 
all their space velocity and therefore cannot radiate. The decay of the meteor into two parts following one after another is visible on the negative. The separated part is visible during two shutter revolutions $(0.08 \mathrm{sec})$. It is also accompanied by a tail.

The light curve of meteor No. 13 is plotted against its altitude in Figure 6. The corresponding tail lengths of this meteor are plotted in the upper part of Figure 6.



Fig. 6. The light curve of meteor No. 13.

The longest tail coincides with the maximum luminosity (the flare). But it is known that at the instant of fragmentation the radiation intensity is a maximum, and the longest tail should have been observed below maximum luminosity, where the displacement of the luminous fragments relative to the parent body is a maximum. There is no displacement of the maximum tail lengths relatively to the meteor flare in Figure 6. Apparently it is necessary to look for an explanation in the persistence of the radiation at a given point, rather than in the displacement of the fragments with respect to the parent body. 
Öpik (1955) assumes that the origin of the luminous wake is connected with recombination of the ions of the meteor substance. Radiative recombination consists of continuous and discrete radiation. Öpik shows that in comparison with discrete radiation the continuous radiation may be neglected, so that on the photographs the radiative recombination spectrum does not differ essentially from the spectrum of neutral atoms excited in consequence of collisions. According to Öpik, efficiency of the discrete radiative recombination for the separate unexcited ion is several times greater than collisional radiation of the separate excited atom. However, Öpik concludes that radiative recombination makes a small contribution to meteor luminescence because: (1) the neutral atom may excite and radiate several times (each ion accounts for several excitations); (2) a small part of the ions survive before recombination; (3) recombination takes place very slowly and its output is small.

We attempt to estimate the contribution of radiative recombination in the meteor tail luminescence. The radiative recombination intensity may be calculated by the formula

where

$$
I=\frac{\tau_{j} \rho_{j} \alpha^{2}}{8 \pi D t} \quad \mathrm{erg} / \mathrm{cm} / \mathrm{sec}
$$

$$
\alpha=\underset{\mu v \mathrm{~d} t}{\beta \mathrm{d} m} \text { electron } / \mathrm{cm}
$$

is linear electron density; $\tau_{j}$, the radiative recombination efficiency; $\beta$ and $\rho_{j}$, the ionization and recombination coefficients respectively; $D$, the diffusion coefficient; $\mu$, the average mass of the atoms of the meteor substance; $\mathrm{d} m / \mathrm{d} t$, the rate of ablation; $t$, the time which is counted from a definite instant, corresponding to the initial formation of the train.

The velocity of meteor No. 39 is $v_{0}=24.6 \mathrm{~km} / \mathrm{sec}$. Its intensity is $I=1 \cdot 12 \times 10^{12}$ $\mathrm{erg} / \mathrm{sec}$. The integration over exposure time gives at $t=0.012 \mathrm{sec}$ the recombination intensity $I=1 \cdot 1 \times 10^{10} \mathrm{erg} / \mathrm{sec}$, which coincides approximately with the intensity measured at this point of the tail $\left(1 \cdot 1 \times 10^{10} \mathrm{erg} / \mathrm{sec}\right)$. For this calculation the usual values of constants were taken: $\tau_{j}=1.5 \times 10^{-12}$ erg for one recombination; $\rho_{j}=2.6 \times 10^{-12}$ $\mathrm{cm}^{3} / \mathrm{sec}$ (Öpik, 1955); $\beta=0 \cdot 2$; and $D=10^{5} \mathrm{~cm}^{2} / \mathrm{sec}$ (Kaščeev and Lebedinec, 1961). The latter value was chosen, with allowance for initial expansion of the train.

It is noteworthy that radiative recombination varies with time comparatively slowly. It decreases considerably slower than the observed intensity of the meteor tail. Apparently the ionization and recombination processes occur in meteor phenomena in a more complex way than according to Equations (4) and (5). In order to investigate these processes it is desirable to take meteor spectra by the method of instantaneous exposure.

The fragmentation of the meteor body into fairly large fragments may be observed by cameras of long focal length. But at large focal lengths the field of view is small, 
and the chance of photographing a meteor decreases. In addition the cross-section distances (i.e. perpendicular to the meteor motion) between fragments are usually so small that even at focal lengths of 1-2 $\mathrm{m}$ they remain below the resolving power of the camera. Longitudinal distances (i.e. coincident with the direction of meteor motion) between fragments increase with time due to differential air resistance, and become larger than the resolving power of the equipment.

For normal photographic observations the images of separate fragments are superimposed one over the other and it is impossible to separate them. The method of instantaneous exposure allows us to separate the images of individual fragments, to investigate the motion of each fragment, and to determine its mass.

Below we give the reduction results of a meteor broken into several fragments. Figure 7 shows some of the instantaneous images of a Perseid (Meteor No. 660 462)
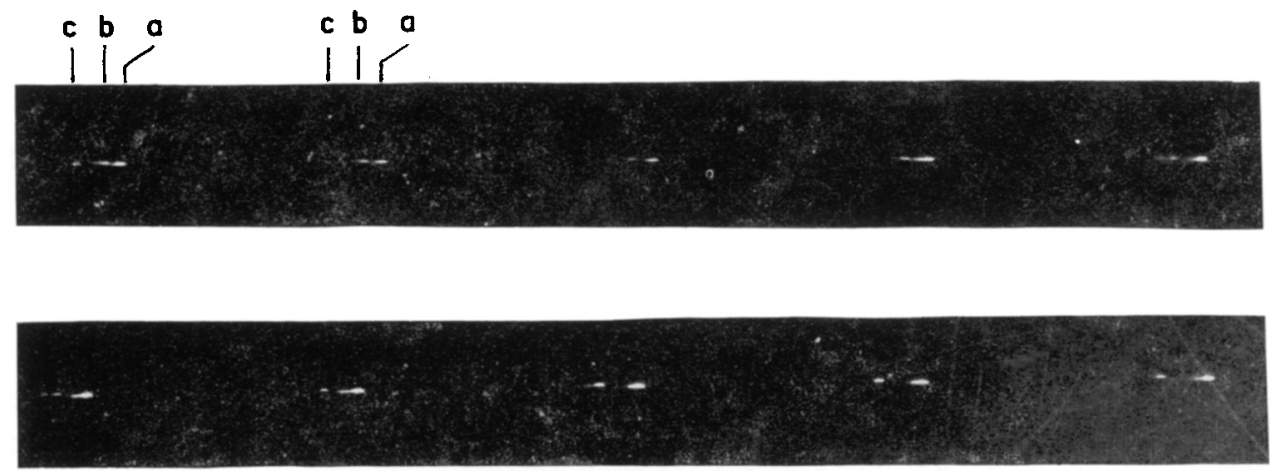

Fig. 7. A series of instantaneous photographs of a bright Perseid of August 12, 1966.

from Dušanbe, August 12, 1966. Its extra-atmospheric velocity and mass were $60.8 \mathrm{~km} / \mathrm{sec}$ and $20 \mathrm{~g}$ respectively. Its duration was about $1 \mathrm{sec}$. The meteor velocity, deceleration and mass are given in Table 5 for various altitudes. This table also gives the values of the ablation coefficient

$$
\sigma=\frac{\ln m_{\infty}^{2}-\ln m^{2}}{v_{\infty}^{2}-v^{2}}
$$

\section{Table 5}

$\begin{array}{ccccccc}H(\mathrm{~km}) & v(\mathrm{~km} / \mathrm{sec}) & \begin{array}{c}\dot{v}_{\text {obs. }} \\ \left(\mathrm{km} / \mathrm{sec}^{2}\right)\end{array} & m(\mathrm{~g}) & \begin{array}{c}\sigma \times 10^{-12} \\ \left(\mathrm{~cm}^{-2} \mathrm{sec}^{2}\right)\end{array} & \begin{array}{c}\dot{v}_{1} \\ \left(\mathrm{~km} / \mathrm{sec}^{-2}\right)\end{array} & \begin{array}{c}\dot{i}_{2} \\ \left(\mathrm{~km} / \mathrm{sec}^{-2}\right)\end{array} \\ 100.5 & 60.7 & -0.332 & 18.4 & 0.49 & -0.238 & -0.691 \\ 97.4 & 60.7 & -0.491 & 16.8 & 0.37 & -0.445 & -0.947 \\ 94.7 & 60.6 & -0.687 & 14.5 & 0.60 & -0.778 & -1.921 \\ 91.6 & 60.5 & -1.012 & 9.2 & 0.89 & -1.714 & -4.203 \\ 88.0 & 60.4 & -1.571 & 3.2 & 6.92 & -4.487 & -10.768\end{array}$


and the theoretical decelerations $\dot{v}_{1}$ and $\dot{v}_{2}$, computed respectively without and with allowance for the reaction of vaporised molecules.

The ablation-coefficient rise is indicative of the progressive fragmentation of the meteor body. On the other hand, the comparison of observed and theoretical deceleration (if we neglect the observational errors) leads to the conclusion that the parent body does not spray, but breaks into several comparatively large fragments. The observational deceleration does not exceed its theoretical value computed for a non-fragmented meteor body. The above conclusion is evident also from the negative value of the fragmentation index for this meteor

$$
\chi=\mathrm{d} s_{\dot{v}_{\text {theor }}}^{\mathrm{d}} \log , \dot{v}_{\mathrm{obs}}
$$

where $s$ is the parameter of mass loss

$$
s=\log \left(\begin{array}{c}
m_{\infty} \\
m
\end{array}-1\right) .
$$

As is seen from Figure 7, the initial parent body breaks into three fragments $a$, $b$ and $c$. Apparently $a$ is the remainder of the parent body; fragments $b$ and $c$ were separated from $a$ at an altitude of about $96 \mathrm{~km}$. Fragment $b$, as more massive, lags behind $a$ less than fragment $c$. In its turn fragment $b$ subsequently loses the first $b^{\prime}$ and then the second $b^{\prime \prime}$ fragments. Two more fragments, $a^{\prime}$ and $a^{\prime \prime}$, were dropped from $a$ at an altitude of about $87-85 \mathrm{~km}$. The images of separate fragments appear after a definite increase of the parent meteor image: the increase of segment $A$ corresponds to the breaking of the fragments $b$ and $c$; segment $\mathrm{B}$ corresponds to the breaking of fragment $b$, etc.

The fragment displacements relative to the parent body $a$ are given in Figure 8 plotted against time. The vertical lines represent the linear sizes of $a$ and $b$. It is clearly visible how the segment increase in length turns into a separation of the images. The luminescence of a fragment begins at once after its separation from the parent body. In this connection it is interesting to estimate the time taken for the 'cold' fragment to reach the intensive vaporisation temperature. For this purpose it is necessary to solve the heat-conductivity equation

at boundary conditions

$$
\frac{\partial T}{\partial t}-\beta^{2}{ }_{\partial x^{2}}^{\partial^{2} T}=\frac{1}{2} \Lambda \rho_{0} v^{3} e^{\kappa t} \frac{\delta(x)}{\rho_{\mathrm{m}} c}
$$

$$
\begin{aligned}
T(0,0) & =T_{0}, \\
T(0, \Delta t) & =T_{\mathrm{v}},
\end{aligned}
$$

where $\beta^{2}=\lambda /\left(\rho_{\mathrm{m}} c\right) ; \kappa=(v \cos Z) / H^{*} ; \lambda$, the heat conductivity; $\rho_{\mathrm{m}}$, the density of meteor body; $c$, the specific heat; $\Lambda$, heat-transfer coefficient; $\rho_{0}$, the air density at the altitude of fragment break; $v$, the meteor velocity; $Z$, zenith distance of the radiant; 


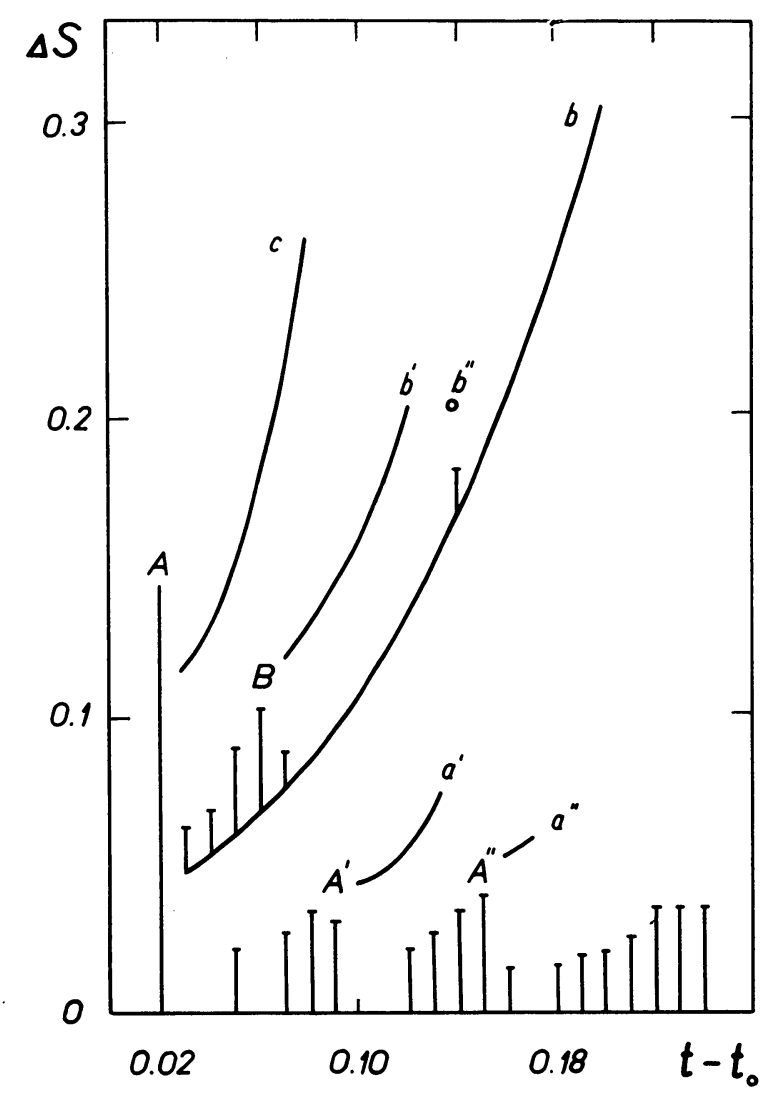

FIG. 8. The fragment displacements relative to the parent body as a function of time. The same meteor as in Figure 7.

$H^{*}$, scale height; and $\delta(x)$, the Dirac delta function which vanishes for $x \doteqdot 0$,

$$
\int_{-\Delta x}^{+\Delta x} \delta(x) \mathrm{d} x=1 .
$$

After the solving of Equation (9) at boundary conditions (10) we obtain the formula that permits us to calculate the time needed to raise the temperature of the meteorbody surface from $T_{0}$ to $T_{\mathrm{v}}$ :

$$
\operatorname{erf}(\sqrt{ } \kappa \Delta t)=\frac{4 \beta \rho_{\mathrm{m}} c \sqrt{v \cos Z}}{\Lambda \rho_{0} v^{3} e^{\kappa \Delta t} \sqrt{H^{*}}}\left(T_{\mathrm{v}}-T_{0}\right),
$$

where $T_{0}$ is the initial temperature of meteor body and $T_{\mathrm{v}}$ is the intensive vaporisation temperature. 
For our meteor we have $\cos Z=0.49, \rho_{0}=10^{-9} \mathrm{~g} / \mathrm{cm}^{3}$ and $H=6.43 \times 10^{5} \mathrm{~cm}$ at the altitude of fragmentation. Assuming $T_{\mathrm{v}}-T_{0}=2000{ }^{\circ} \mathrm{K}$ with $\Lambda=1$ and $\Lambda=0 \cdot 1$ we obtain respectively $\Delta t=0.009 \mathrm{sec}$ and $\Delta t=0.23 \mathrm{sec}$. During $0.009 \mathrm{sec}$ the fragment in the 'cold' state could not lag appreciably behind the parent meteor body. But $\Delta t=0 \cdot 23$ sec is sufficiently long to detect the fragment lag. During this time the fragment would lag behind the meteor body without radiation. This means that its corresponding images would appear suddenly at some distance from the leading meteor body, a fact not actually observed.

The progressive lengthening of the corresponding meteor-body tail, and its transition to a separate image, is seen from Figures 7 and 8 . Therefore, if we assume that fragments break 'cold' we can conclude that $\Lambda \simeq 1$.

In addition, it may be shown that at the moment of fragmentation the meteor body was moving under conditions close to free molecules. We calculate the Knudsen number

$$
K n=\frac{l}{d_{0}},
$$

where $l$ is the free path of the molecules; $d_{0}$, the peculiar cross-section of the meteor body.

At the altitude of the first fragmentation (about $90 \mathrm{~km}$ ) $l=8 \cdot 16 \mathrm{~cm}$ (Reference Book on Geophysics, 1965) and $d=2.8 \mathrm{~cm}$ (if the density of the meteor body is $\rho_{\mathrm{m}}=1 \mathrm{~g} / \mathrm{cm}^{3}$, see Table 1). $K n=3 \cdot 1$, i.e. it is larger than the critical value $K n=2$ (Devienne, 1958), which corresponds to the transition from slip conditions to free-molecular flow conditions. For the 'normal' density $\left(3 \cdot 5-7 \cdot 8 \mathrm{~g} / \mathrm{cm}^{3}\right)$ of a meteor body $K n>4 \cdot 5-6$, which also corresponds to free-molecular flow.

Since the meteor-body motion takes place in free-molecular conditions, two forces act on the frontal surface of a meteor body: (1) hydrodynamic pressure of impacting air molecules, and (2) reaction force arising as a result of the meteor vaporization. At the disintegration altitude the first force is equal to $\pi r^{2} \rho v^{2}=1.9 \times 10^{5}$ dynes and the second one is $-\frac{4}{9}(\mathrm{~d} m / \mathrm{d} t) v_{t} \simeq 2 \times 10^{6}$ dynes $\left(v_{t}\right.$, the thermal velocity of vaporized molecules). Only highly fragile meteor bodies will disintegrate under the influence of these forces. Fragile deformation also depends on the amount of energy absorbed per unit time. By intensive vaporization the external energy is spent completely in ablation of the surface layer. This energy barely penetrates inside.

Jones and Kaiser (1966) assume that at high altitudes the meteor bodies become fragile as a result of the thermal shock when they warm up suddenly. This may be even before melting. These authors used equations with the help of which they calculated the elastic stresses developed by thermal shock inside the meteor body. But they do not show that such a thermal shock really occurs. In the case of meteor No. 660462 , if splitting of the meteor body appeared before surface-layer melting, then the meteor should have disintegrated at a higher altitude and at the beginning 
of the intensive vaporization, as is the case for faint meteors. In fact the fragmentation occurred more than $0.5 \mathrm{sec}$ after the meteor appeared. But as was shown by Levin (1956), and this was emphasized above, during intensive vaporization all energy is spent for vaporization and the meteor body remains cold except for a thin surface layer. Moreover, inside the meteor body the temperature gradient is so negligible that it cannot cause disintegration.

Usually disintegration of a meteor body into several fragments occurs after a meteor flare occasioned by a sudden vaporization of a melted layer. Such rapid vaporization produces a split wave directed inside the meteor body, and in turn this wave may cause the breaking of fragments from the main part of the meteor body (Kolsky, 1953).

The foregoing examples show that meteor phenomena have a complex nature. For their successful investigation in the future it is necessary to develop research by the method of instantaneous exposure, together with other methods.

\section{References}

Babadžanov, P. B., Kramer, E. N. (1965) Astr. Zu., 42, 660.

Devienne, M. (1958) Frottement et échanges thermiques dans les gaz raréfiés, Paris.

Halliday, I. (1958) Astrophys. J., 127, 245.

Jacchia, L. G., Verniani, F., Briggs, R.E. (1965) Smithson. Astrophys. Obs. Spec. Rep., 175.

Jones, J., Kaiser, T.R. (1966) Mon. Not. R. astr. Soc., 133, 411.

Kaščeev, B. L., Lebedinec, V.N. (1961) Radar Investigations of Meteor Phenomena, Publ. House Acad. Sci. USSR, Moscow.

Kolsky, H. (1953) Stress Waves in Solids, Oxford.

Levin, B.Ju. (1956) The Physical Theory of Meteors and Meteoric Matter in the Solar System, Publ. House Acad. Sci. USSR, Moscow.

Levin, B.Ju. (1961) Bjull. Kom. Komet. Meteor., 6, 3.

McCrosky, R.E. (1958) Astr. J., 127, 245.

Öpik, E.J. (1955) Proc. R. Soc., 230, 463.

Reference Book on Geophysics (1965) Publ. House 'Science', Moscow.

\section{DISCUSSION}

Ceplecha: Congratulations on the results. I would be very happy, if grating spectrographs with a rotating shutter for instantaneous exposures could be used for meteors in future work.

Babadžanov: In 1966 the first spectrum of a meteor was obtained by the method of the instantaneous photograph, but it has not yet been analyzed. 